\title{
A Formal Machine Learning or Multi Objective Decision Making System for Determination of Weights
}

\author{
Ganesan.R \\ Department of C.S.E \\ Bharath University \\ Chennai, India
}

\author{
Shanmuga Priyan.P \\ Department of C.S.E \\ Bharath University \\ Chennai, India
}

\author{
Kerana Hanirex.D \\ Dept. of C.S.E \\ Bharath University \\ Chennai, India
}

\begin{abstract}
Decision-making typically needs the mechanisms to compromise among opposing norms. Once multiple objectives square measure is concerned of machine learning, a vital step is to check the weights of individual objectives to the system-level performance. Determinant, the weights of multi-objectives is associate in analysis method, associated it's been typically treated as a drawback. However, our preliminary investigation has shown that existing methodologies in managing the weights of multi-objectives have some obvious limitations like the determination of weights is treated as one drawback, a result supporting such associate improvement is limited, if associated it will even be unreliable, once knowledge concerning multiple objectives is incomplete like an integrity caused by poor data. The constraints of weights are also mentioned. Variable weights square measure is natural in decision-making processes. Here, we'd like to develop a scientific methodology in determinant variable weights of multi-objectives. The roles of weights in a creative multi-objective decision-making or machine-learning of square measure analyzed, and therefore the weights square measure determined with the help of a standard neural network.
\end{abstract}

Keywords: Decision Making, Machine Learning, Data Integrity, Optimization, Constraints.

\section{INTRODUCTION}

System Science typically considers the knowledge domain interactions among a group of subsystems in individual disciplines as a result of associate rising trend and therefore the desires of finding out ever increasingly advanced systems in engineering, society, setting, and science, intensive analysis works are performed within the field of system science. The careful discussions and basic ideas during this field will be found in several literatures. As an example, Warfield stressed that system science could be a important conception with the potential to serve several functions and comprehend several things in its view. Bailey mentioned the history of the event of system science and known 10 goals together with 10 challenges; among these challenges, 3 square measure associated with the contradictions caused by multiple disciplines. Jamshidi created his remarks that system optimizations, reliable and strength among a group of subsystems to realize a standard system goal became the main focus of the many applications like region, automotive, military, environmental, and repair business. It's fascinating to realize activity from subsystems for associate optimized performance at a high system level. It's potential to elucidate, predict, and formulate the decision-making issues of advanced systems

\section{LITERATURE SURVEY}

Modular Neural Networks (MNNs) could be a quickly growing field in artificial Neural Networks (NNs) analysis.[8] This paper surveys the various motivations for making MNNs: biological, psychological, hardware, and machine. Then, the final stages of MNN style square measure printed and surveyed likewise, viz., task disintegration techniques, learning methods and multi-module decision-making ways. Benefits and downsides of the surveyed strategies square size known, secondary degreed an calculation with position to sensible possible is delivered. Finally, some general recommendations for future styles square measure conferred.

Option evaluation with standard Neural Networks investigates a statistic standard neural network (MNN) model to cost the
European decision choices. The modules square measure supported time to maturity and moneyless of the choices. The choice value perform of interest is homogenized of degree one with reference to the underlying index value and therefore the strike value. Compared to associate degree array of constant quantity and statistic models, the MNN methodology systematically exerts superior out-of-sample evaluation performance. We tend to conclude that modularity improves the generalization properties of ordinary feed forward neural network choice evaluation models

State of the Art and Future Trends in Distributed Systems and present Computing summarizes trends in communication paradigms for distributed systems, mentions established and new computer code infrastructures for distributed systems (such as CORBA and Jini), and provides an summary of mobile code and mobile agent principles. It then explains the vision of present Computing and future networked sensible devices together with techniques like RFID tags (or "smart labels"). It conjointly discusses problems in spontaneous networking, service discovery, and connected ideas. The report quotes munificently from variety of on-line resources found on the net.

Pattern classification has been with success applied in several drawback domains, like biometric recognition, document classification or diagnosing. Missing or unknown knowledge square measure a typical disadvantage that pattern recognition techniques have to be compelled to agitate once determination real-life classification tasks. Machine learning approaches and strategies foreign from applied math learning theory are most intensively studied and employed in this subject. The aim of this work is to research the missing knowledge drawback in pattern classification tasks, and to summarize and compare a number of the well-known strategies used for handling missing values.

[8][1]We propose a unique 2-stage soft computing approach for knowledge imputation, involving native learning and world approximation in bicycle, whereas within the literature just one of them is employed. In stage 1, K-means formula is employed 
to switch the missing values with cluster center. Stage two refines the resultant approximate values victimization multilayer perception (MLP). MLP is trained on the whole knowledge with the attribute having missing values because the target variable one at a time. The hybrid is tested on two benchmark issues every in classification and regression victimization 10 -fold cross validation. Altogether datasets, some values, that square measure indiscriminately removed, square measure treated as missing values. The particular and therefore the foretold values obtained square measure compared by victimization Mean Absolute share Error (MAPE). we tend to observe that, the MAPE price is reduced from stage one to stage two, indicating the hybrid approach resulted in higher imputation compared to stage one alone

[6]Ensembles of learning machines represent one among the most current directions in machine learning analysis, and are applied to a large vary of real issues.[10] Despite of the absence of associate degree united theory on ensembles, there square measure several theoretical reasons for combining multiple learners, associate degreed an empirical proof of the electiveness of this approach.[8] During this paper we tend to gift a short summary of ensemble strategies, explaining the most reasons why they're able to outmatch any single classifier at intervals the ensemble, and proposing taxonomy supported the most ways that base classifiers may be generated or combined along.

the Analytic Hierarchy method is associate degree introduce particle to the Analytic Hierarchy method[2][1] A multi criteria deciding approach within which factors square measure organized during a class-conscious structure. The principles and therefore the philosophy of the idea square measure summarized giving general background informant particle of the sort of menstruation utilized, its properties and applications.

One of the crucial issues in \{decision making |deciding |higher cognitive method \} process is to assess the relative importance or weights of various attributes. [3] This paper presents associate degree objective methodology to work out relative criteria weights that relies on co relational analysis particularly principal elements analysis. Consistent with the methodology, delineate during this paper, there's a clear stage to work out weight coefficients from Kaiser-Meyer-Olkin live and from square issue loadings.

While gauging the performances of in operation entities victimization inexact data on the input and output importance weights, associate degree entity is taken into account Farrell economical as long because it outperforms its peers for a minimum of one possible combination of the weights for inputs and outputs. [4]This paper argues that Farrell potency computations square measure supported associate degree optimistic perspective and a Farrell-efficient entity might perform rather poorly once weights similar to realistic issues square measure allotted to inputs and outputs.[7] Associate degree entity is outlined as sturdy economical if its relative potency score reaches one altogether possible combos of the input and output weights. A applied mathematics based mostly approach is projected to perform what's spoken as sturdy potency analysis to spot sturdy economical entities. In distinction to Farrell potency analysis, sturdy potency associate degree analysis involves the computation of all-time low potency score which will be allotted to an entity relative to the best score among all the entities wherever the same combination of weights for inputs and outputs is applied. the assembly risk set underlying the projected approach is additionally outlined and understood. Associate degree experimental study illustrates that compared with Farrell potency analysis sturdy potency analysis has scammer discrimination capability and therefore the entity it identifies as economical has superior average performance.

[9]Business intelligence (BI) is that the method of gathering enough of the proper data within the right manner at the proper time, and delivering the proper results to the proper individuals for decision-making functions in order that it will still yield real business advantages, or has a positive impact on business strategy, tactics, and operations within the enterprises. [5] This paper was supposed as a brief introduction to the study of business intelligence in enterprise computing atmosphere. Additionally, the conclusions illustrate the challenges to broad and deep readying of business intelligence systems, and supply the proposals of constructing business intelligence more practical.

\section{PLANNED SYSTEM}

The formulation of the decision-making method of advanced systems has been mentioned and therefore the focus is directed on the way to assess relative importance once multiple criterion square measures is concerned. The relative importance of one type criterion over another is bestowed by weights. It's been found that the determination of weights involves associate analysis method, that shouldn't be merely treated as associate improvement. a scientific methodology has been planned to see the weights for multiple objectives befittingly. The discussion on consistency at the tip reveals that the weights don't seem to be essentially consistent once variable weights square measure applied and synthesized within the method of determination.

\section{MULTI-OBJECTIVE DECISION MAKING}

Multi-Objective Decision Making is a technique for ensuring the integrity of data in outsourcing storage service. It allows a client that has stored data at an un-trusted server to verify that the server possesses the original data without regaining it. The model gives out probabilistic evidences of possession by sampling random sets of blocks from the server, which radically cuts $\mathrm{I} / \mathrm{O}$ expenses. The customer preserves a continuous amount of metadata to check the proof. The test/reaction protocol conveys a small, continuous amount of data, which minimizes network communication. Thus, the model for remote data checking supports large data sets in widely-distributed storage systems. We are quite aware about square measure of single objective call issues from our previous study of applied mathematics and alternative improvement fields like inventory management, and project management. However, several call things incorporate deciding once over one objective has to be thought of (called multi-objective call making). Such associate approach is typical to investment issues wherever business banks have to be compelled to balance come and risk.

\section{MACHINE LEARNING}

Machine learning deals with the problem of the way to build pc programs that improve their performance at some tasks through expertise. Machine learning algorithms have evidenced to be of nice sensible worth in an exceedingly sort of application domains. Not amazingly, the sphere of software system engineering seems to be a fertile ground wherever several software system development and maintenance tasks can be 
developed as learning issues and approached in terms of learning algorithms. This book deals with the topic of machine learning applications in software system engineering. It provides a summary of machine learning, summarizes the stateof-the-practice, provides a classification of the present work, and offers some application pointers.

\section{MODULAR NEURAL NETWORKS (MNN)}

Modular neural networks, as combined structures, have conjointly a biological background: Natural neural systems square measure composed of a hierarchy of networks designed of parts specialized for various tasks. In general, combined networks square measure a lot of powerful than flat unstructured ones.

In this manner we tend to attain the conception of standard neural networks. many general problems have crystal rectifier to the event of standard systems.

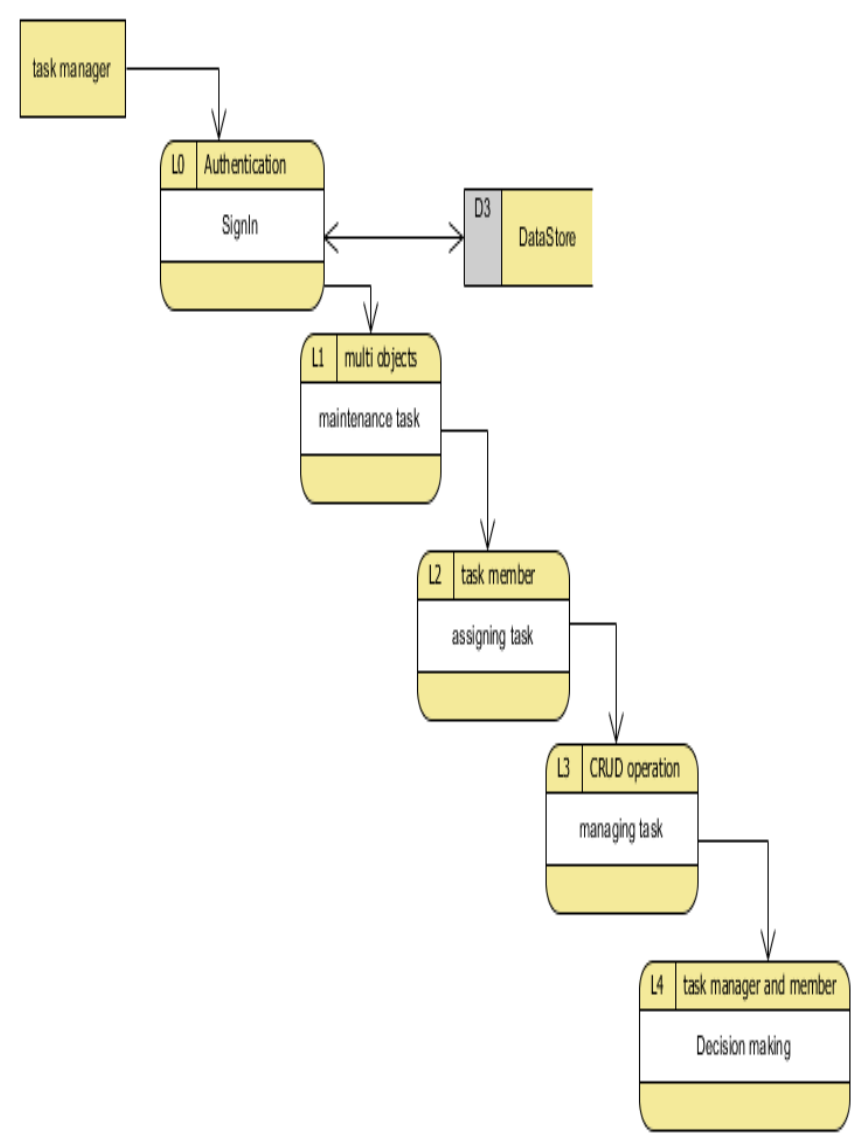

Figure.1 data flow diagram

- $\square$ Incorporating knowledge: Complete modules square measure associate degree extension of the approach mentioned in Sect. 10.3.5 of learning with hints.

- $\square$ Data merging and prevision averaging: Committees of networks may be thought-about as composite systems fabricated from similar parts.

- $\square$ Combination of techniques: quite one methodology or category of network may be used as building block.
- $\square$ Learning totally different tasks simultaneously: Trained modules are also shared among systems designed for various tasks.

- $\square$ Robustness and instrumentality: The combined network will grow step by step and may be created fault-tolerant.

\section{CONCLUSION}

The formulation of the decision-making method of complicated systems has been mentioned and therefore the focus is directed on the way to valuate relative importance once multiple criterion square measures concerned. The relative importance of 1 style criterion over another is conferred by weights. it's been found that the determination of weights involves associate degree analysis method, that shouldn't be merely treated as associate degree optimization. a scientific methodology has been projected to work out the weights for multiple objectives befittingly. The discussion on consistency at the top reveals that the weights don't seem to be essentially consistent once variable weights square measure applied and synthesized within the method of determination. The analysis of weights for multi-objective deciding could be a crucial issue in several applications like MODM and MFML. solely the load determination and consistency square measure centered within the conferred work

\section{ACKNOWLEDGMENTS}

The author would like to thank the Vice Chancellor, DeanEngineering, Director, Secretary, Correspondent, HOD of Computer Science \& Engineering, Dr. K.P. Kaliyamurthie, Bharath University,Chennai for their motivation and constant encouragement. The author would like to specially thank Dr. A. Kumaravel, Dean, School of Computing, Bharath University for his guidance and for critical review of this manuscript and for his valuable input and fruitful discussions in completing the work and the Faculty Members of Department of Computer Science \&Engineering. Also, he takes privilege in extending gratitude to his parents and family members who rendered their support throughout this Research work.

\section{REFERENCES}

[1] G. Valentini and F. Masulli, "Ensembles of learning machines," in Proc. WIRN VIETRI, M. Marinaro and R. Tagliaferri, Eds., 2002.

[2] C. Wang, "Advances in information integration infrastructures supporting multidisciplinary design optimisation," Enterprise Inf. Syst., Aug. 2012

[3] Y. M. Wang and J. K. Zhang, "A method based on standard and mean deviations for determining the weight coefficients of multiple attributes and its applications," Appl. Stat. Manage., 2003

[4] P. Wang, S. Feng, and R. Zhang, "Method integration and variable weight synthesis in solving hybrid objective systems," Sep. 2008.

[5] X. Wang and X. Xu, "DIMP: An interoperable solution for software integration and product data exchange," Enterprise Inf. Syst., Aug. 2012 
International Journal of Computer Applications Technology and Research

Volume 4- Issue 4, 287 - 290, 2015, ISSN:- 2319-8656

[6] Q. Liu and C. Wang, "Multi-terminal pipe routing by Steiner minimal tree and particle swarm optimization," Aug. 2012

[7] W. Q. Liu, "The ordinary variable weight principle and multiobjective decision making," Mar. 2000.

[8] X. B. Lam, Y. S. Kim, A. D. Hoang, and C. W. Park, "Coupled aerostructural design optimization using the Kriging model and integrated multiobjective optimization algorithm," J. Optim., Sep. 2009.

[9] L. Li and J. Liu, "An efficient and flexible web-servicesbased multidisciplinary design optimization framework for complex engineering systems," Aug. 2012.

[10] Y. J. Pang and K. D. Liu, "The consistency check in the analytic hierarchy process is not necessary condition of sequencing," J. Hebei Inst. Arch. Sci. Technol, 2002. 